\title{
Microquasars: Open Questions and Future Perspectives
}

\author{
I.F. Mirabel \\ CEA/DSM/SAp. CE-Saclay, France \& IAFE/CONICET, Argentina.
}

November 12, 2000

\begin{abstract}
The discovery and subsequent study of microquasars lead to major progress in our understanding of: 1) the nature of relativistic jets seen elsewhere in the universe, and 2) the connection between the accretion onto compact objects and the formation of collimated jets. A detailed account of the major progress accomplished until present was published in Annual Review of Astronomy \& Astrophysics (Mirabel \& Rodríguez, 1999). Here I review the questions that remain unanswered, as well as the future perspectives that this new field of research is opening.
\end{abstract}

Keywords: X-rays:stars, X-ray binaries, superluminal motion, microquasars

\section{Major progress accomplished}

In relativistic sources located in the Milky Way, two-sided moving jets can be observed, and therefore can be overcome several of the ambiguities that had dominated the physical interpretation of extraglactic jets, where so far only the motions of one-side of the jets could be followed. From the observation of two-sided moving jets in microquasars, the system of equations can be solved. For the first time, an upper limit of the distance to a microquasar was derived from the proper motions using special relativity constraints (see Mirabel \& Rodríguez 1999).

Major progress has also been made in the understanding of the accretion/ejection phenomenology. From multiwavelength observations, the connection between accretion flow instabilities -observed in the X-rayswith the ejection of relativistic plasma -observed at radio, infrared, and possibly optical and X-ray wavelengths-, has been established on a firm basis. After the softer component of the X-ray emitting plasma disappears, the inner accretion disk is rapidly re-established and the plasma that produces the hard X-ray component, is blown away (Mirabel et al. 1998) in the form of collimated relativistic jets (Dhawan, Mirabel \& Rodríguez, 2000).

The X-ray source GRS 1915+105 (Castro-Tirado et al. 1994) has become a main target to study accreting black holes of stellar mass. However, the discovery and study of other microquasars is important not only to enhance the statistical sample, but also to make progress on the different aspects of the diverse phenomenology that each new object of this class has revealed (Mirabel \& Rodríguez 1999). 


\section{Open questions}

\section{What is a microquasar ?}

By this term we usually design stellar-mass black holes that mimic, on a smaller scale, many of the phenomena seen in quasars. The question is whether under this concept we should also include neutron star systems with jets. Although the physical meaning of the quasarmicroquasar analogy as proposed by Mirabel \& Rodríguez (1998) is valid for black holes, because in many cases the nature of the compact object is unknown, we may currently include in this subclass of stellar sources, neutron star binaries that produce relativistic jets.

Are all accreting black holes of stellar mass microquasars ?

All microquasars are accreting compact objects of stellar mass, but is the reverse true ? This question can be re-formulated as follows: do all X-ray black hole binaries produce jets ? From the theoretical point of view jets are needed to liberate angular momentum. On the other hand, observations show that X-ray emitting black holes, in addition to possible synchrotron emission associated to sporadic outbursts, always exhibit flat-spectrum compact counterparts of synchrotron emission. It has been proposed (Fender, 2000) and demonstrated by high resolution images (Dhawan, Mirabel \& Rodríguez, 2000), that these flat spectrum compact radio counterparts are thick collimated jets of AU size scales. Furthermore, from multiwavelength observations at radio, infrared, and X-ray wavelengths of the large-scale jets in SS433/W50, we know that in some circumstances jets exist without being seen. Therefore, the answer to this question is that probably, all accreting black holes of stellar mass are microquasars.

Are the Lorentz factors of the bulk motions in the jets from quasars different from those in microquasars?

In other words, is the quasar-microquasar analogy valid? The microquasar jets observed so far have bulk speeds that are statistically smaler than in quasars. For instance, in quasars are found jets with bulk speeds of up to $\sim 20$ c, but no microquasar with such jets has so far been found. Is this due to some fundamental difference that would invalidate the physical analogy between quasars and microquasars, or is it rather due to selection effects related to the Doppler favoritism needed in order to measure the proper motion of the ejecta in distant quasars ? Although from the observational point of view this remains an open question, theoretically it is not clear why the bulk motion in jets from supermassive black holes would be different from that in the jets from stellar mass black holes. 
Could the terminal velocity of the jet be a diagnostic for the nature of the collapsed object (NS versus $\mathrm{BH}$ ) ?

In all areas of astrophysics there have been increasing evidences that accretion is always related to the production of collimated jets. The observed outflow speeds seem to be of the order of the escape velocity from the surface of the accreting object. Objects as diverse as very young stars, nuclei of planetary nebulae, and accreting white dwarfs have jets with non-relativistic velocities $\left(\sim 100-10000 \mathrm{~km} \mathrm{~s}^{-1}\right)$, whereas neutron stars and black holes produce jets with relativistic speeds $V \geq 0.1 c$ (Livio, 1999; Mirabel \& Rodríguez, 1999). If the present trends between the escape velocities from the accreting objects and the velocity of the outflows were confirmed as a strong correlation, it would imply that gravity is important and dominates over MHD mechanisms (Meier et al. 1997). However, it is not clear whether we will be able to discriminate between neutron stars and black holes by knowing the terminal velocity of the collimated outflows. For instance, Sco X-1 which problably contains a neutron star, has lobes moving with an average velocity of 0.45 c (Fomalont, Geldzahler \& Bradshaw, 2000). Since only a handful of relativistic jet sources have been discovered so far in the Galaxy (Mirabel \& Rodríguez, 1999), we need the discovery and study of more sources to obtain a statistically significant number to answer this question.

Why are QPOs in microquasars and not in AGNs ?

The scale of time of the instabilities in the accretion disk of black holes is proportional to the mass of the black hole. QPOs with periods of $\sim 1$ sec in black holes of $10 \mathrm{M}_{\odot}$ would correspond to QPOs of $\sim 1$ day to $\sim 3$ years in black holes with masses of $10^{6}$ and $10^{9} \mathrm{M}_{\odot}$ respectivelly. QPOs of $1 \mathrm{sec}$ in microquasars correspond to typical fluctuations of $\sim 5 \%$ of the X-ray flux. At X-rays the companion binary stars are much dimmer than the accretion disk. On the other hand, the accretion disk in AGNs is cooler (Mirabel \& Rodríguez, 1999), and QPOs should be observed in the UV and optical, wavelengths at which stars mostly radiate, and interstellar absorption is important. Since AGNs may be blurred by nuclear starbursts, it may be difficult to detect fluctuations of $\sim 5 \%$ from AGNs that are embedded in starburst nuclei.

One then may ask about the analogous of the large scale oscillations of the flux $(\geq 50 \%)$ with periods of $\sim 30$ min regularly observed in GRS 1915+105 (Greiner, Morgan \& Remillard, 1996). In black holes with masses in the range of $10^{6}$ to $10^{9} \mathrm{M}_{\odot}$ analogous QPOs would correspond to periods in the range of 5 to 500 years, which are difficult to monitor in human time-scales (Sams, Eckart \& Sunyaev, 1996). 
To image the jet close to the black hole, do microquasars offer an advantage with respect to AGNs ?

No. The Schwarzschild radii of BHs are proportional to their mass and in these units nearby AGNs can be imaged closer to the BH than microquasars. For instance, M87 which is in the Virgo cluster at a distance of $15 \mathrm{Mpc}$ and contains a $\mathrm{BH}$ of $310^{9} \mathrm{M}_{\odot}$ has been imaged with a resolution of $\sim 50$ times the Schwarzschild radius (Junor, Biretta \& Livio, 1999). A given angular size projected on the supermassive BH of M87 is in terms of its Schwarzschild radius $10^{4}$ smaller than the same distance projected on a $\mathrm{BH}$ of $10 \mathrm{M}_{\odot}$ at a distance of $2 \mathrm{kpc}$ from the Sun. Therefore, relative to supermassive BHs in the Local Universe, the study of stellar mass BHs presents an advantage because of the time scales, but not as far as the dimensions of length are concerned.

What is the connection between accretion and ejection ?

In GRS 1915+105 large amounts of X-ray emmiting plasma $\left(\sim 10^{6}\right.$ $\left.\mathrm{L}_{\odot}\right)$ disappear in less than a few seconds and soon after synchrotron jets are formed (Fender \& Pooley, 1998; Eikenberry et al. 1998; Mirabel et al. 1998). Recent analysis shows that the X-ray flux that suddenly disappears only corresponds to the softer component $(2-20 \mathrm{keV})$. The formation of the jets starts later, at the time of a spike that consists of a sudden increase in the flux of the soft component simultaneous with a decrease of the flux of the harder component $(20-60 \mathrm{keV})$. In the context of current models these observations imply that the inner accretion disk first disappears (Belloni et al. 1997), and when the inner accretion disk is being re-established, a shock is produced which triggers the blow up of the plasma that was emmiting the hard X-rays in the form of collimated jets at relativistic speeds (Dhawan, Mirabel \& Rodríguez, 1999). In GRS 1915+105, an analogous correlation between the sudden disappearance of the 20-100 keV measured by BATSE and major ejection events seen in the radio with the VLA had been observed (see Mirabel \& Rodríguez, 1999 for references and the discussion of this issue).

What fraction of the inflow goes into the jets?

The observations described above indicate that the matter and energy emitting in the soft X-rays that suddenly disappears is perhaps advected into the $\mathrm{BH}$, since it is not immediately translated neither in hard X-rays nor in synchrotron jets. Because of the uncertainties in estimating the mass of the X-ray emitting plasma it is difficult to estimate the fraction of the inflow that goes into the jets. On the contrary, assuming equipartition, the amount of mass and energy of the jets can be calculated (Fender \& Pooley, 1998; Mirabel et al. 1998). 
Now there are increasing evidences that the power of the jets may be a large fraction of the accretion power. For instance, in its very high state, GRS 1915+105 may have a short-term jet power of $\sim 10^{39} \mathrm{erg} \mathrm{s}^{-1}$ (Mirabel \& Rodríguez, 1999; Fender, 2000), which is a large fraction of the observed accretion power. This is consistent with the model for a rapidly rotating $\mathrm{BH}$ with a high accretion rate by Meier (2000).

What is the mechanism that launches the jets ?

MHD power which was nicely reviewed by Meier (2001) and may play an important role. However, it is still unclear the relative importance of the rotating thin accretion disk mechanism by Blandford \& Payne (1982) and that from the frame-dragged accreting matter inside the ergosphere of a rotating BH (Blandford \& Znajek, 1977). The X-ray spectral properties of microquasars with powerful jets (e.g. GRS 1915+105, GRO J1655-40) indicate that these are rotating BHs with spins near maximum (Zhang, Cui \& Chen, 1997), which is consistent with the Blandford \& Znajek mechanism. On the other hand, the Blandford \& Payne mechanism is consistent with the following two recent observations: 1) the large opening angle of $\sim 60^{\circ}$ of the jet in M87 (Junor, Biretta \& Livio, 1999) which seems to be the two dimensional image of a jet with a magnetic polar field angle $\geq 30^{\circ}$. The M87 jet strongly collimates at 30-100 Schwarzschild radii $\left(\mathrm{r}_{s}\right)$ from the $\mathrm{BH}$, collimation continuing out to $\sim 1000 \mathrm{r}_{s}$. 2) The semicontinuous emanation of the jets in the infrared and radio during time intervals of the order of minutes, as observed in GRS 1915+105 (Eikenberry et al. 1998; Fender \& Pooley, 1998; Mirabel et al. 1998).

Although several numerical simulations have been made, there are still no definitive tests to discriminate the relative role of these two MHD mechanisms.

What are the QPOs of maximum fix frequency ?

Because QPOs with maximum fix frequency have been observed many times in some microquasars (e.g. $67 \mathrm{~Hz}$ in GRS 1915+105; Morgan, Remillard \& Greiner, 1997), it is believed that they are related to fundamental properties of the BHs, such as its mass and spin. The problem is that there are more than 3 different alternative explanations in terms of General Relativity (see Mirabel \& Rodríguez, 1999 for references). The theories should provide the observational tests that would discriminate among the models proposed.

Are the jets discrete plasmons or semi-continuous flows with internal and/or external shocks?

Internal shock models of microquasar jets (Bodo \& Ghisellini, 1995; Kaiser, Sunyaev \& Spruit, 2000) propose that the plasma velocity is smaller than the pattern velocity. Outbursts in the core lit up the 
jets are by shock fronts that travel along the jets which accelerate the relativistic particules that emit the synchrotron radiation. Internal shock models relax the requirements on the power of the central engine because much of the energy underlying the outbursts is stored in the continuous jet. An alternative model of the twin moving radio lobes observed in Sco X-1 (Fomalont, Geldzahler \& Bradshaw, 2000) is that they are intetraction of the energy flow from beams with the interstellar medium. The lobes advance at $\sim 0.45 \mathrm{c}$ but the beam velocity is $\geq 0.95 \mathrm{c}$. While the observations of GRS 1915+105 and SS433 would be more consistent with the internal shock model, the moving lobes in Sco X-1 seem to be shocks in working surfaces of an external medium. While the observed velocities of the ejecta in GRS 1915+105 $(\sim 0.95 \mathrm{c})$ and SS $433(\sim 0.26 \mathrm{c})$ have been the same over several years, whereas in Sco X-1 the speeds for different pairs of components at different times range between $0.31 \mathrm{c}$ and $0.57 \mathrm{c}$. This suggests that different physical processes may dominate in different sources.

Jets in microquasars, $\gamma$-ray burst (GRB) afterglows and AGN show analogous phenomenologies. It is interesting that the internal shock model originally developed for GRBs (Mészáros \& Rees 1997) and the external shock model originally proposed for the terminal lobes in AGNs (Blandford \& Rees, 1974) are now being applied to microquasars (Kaiser, Sunyaev \& Spruit 2000; Fomalont, Geldzahler \& Bradshaw, 2000). Conversely, the plasmon model originally proposed to interpret the microquasar jets is being proposed in the "cannonball" model of GRBs by Dar \& De Rújula (2000).

\section{Have extragalactic microquasars been identified ?}

Ultraluminous X-ray compact sources have been identified in several nearby spiral galaxies (Colbert \& Mushotzky, 1999; Makishima et al. 2000 ) as well as in dwarf galaxies (Mirioni \& Pakull, 2000). These enigmatic sources have luminosities in the range of $\mathrm{L}_{x}=10^{38-40} \mathrm{erg} \mathrm{s}^{-1}$, and it has been proposed by Colbert \& Mushotzky (1999) that they are BHs of $10^{2-4} \mathrm{M}_{\odot}$. Makishima et al. (2000) propose that some of these superluminous sources are BHs as the microquasars GRS 1915+105 and GRO J1655-40, with masses below $\sim 100 \mathrm{M}_{\odot}$ and high disk temperatures because rapid rotation gets the disk closer to the $\mathrm{BH}$, hence hotter. Unfortunately, the VLBA array does not have the sensitivity to image in its plateau state extragalactic "super-microquasars" as GRS $1915+105$.

Why superluminal microquasars have unbroken power law photon spectra?

Grove (1999) has shown that the microquasars with superluminal jets have power-law-gamma-ray states with an index of 2.5-3. Con- 
trary to Cygnus X-1 and 1E1740.7-2942, the superluminal sources GRO J1655-40 and GRS 1915+105 have unbroken power laws up to almost $1 \mathrm{MeV}$ with no indication of Comptonization. A similar photon spectrum has been observed by SAX in XTE 1118+48 (Frontera et al. 2001). Since we don't know what is the origin of the electrons that produce the gamma-ray photons it is still unclear why some BHs would have broken, whereas others have unbroken power law photon spectra. The sensitivity of INTEGRAL in this domain of energy will certainly provide new perspectives on this issue.

\section{Will microblazars be found ?}

In most microquasars where $\theta$ (the angle between the line of sight and the axis of ejection) has been determined, large values are found $\left(\theta \geq 70^{\circ}\right)$. Namely, except some exceptions (as in the case of Sco X-1 where $\theta=45^{\circ}$; Fomalont, Geldzahler \& Bradshaw, 2000), the axis of ejection in most cases is close to the plane of the sky. This is consistent with the statistical expectation since the probability of finding a source with a given $\theta$ is proportional to $\sin \theta$. We then expect to find as many objects in the $60^{\circ} \leq \theta \leq 90^{\circ}$ range as in the $0^{\circ} \leq \theta \leq 60^{\circ}$ range. However, this argument suggests that we should eventually detect objects with a small $\theta$. For objects with $\theta \leq 10^{\circ}$ we expect the timescales to be shortened by $0.5 \gamma^{2}$ and the flux densities to be boosted by $8 \gamma^{3}$ with respect to the values in the rest frame of the condensation. For instance, for motions with $v=0.98 \mathrm{c}(\gamma=5)$, the timescale will shorten by a factor of $\sim 50$, the flux densities will be boosted by a factor of $\sim 10^{3}$, and the photon spectrum of the source will be very hard. Then, for a galactic source with relativistic jets and small $\theta$ we expect fast and intense variations in the observed flux. Microblazars may be quite hard to detect in practice, both because of the low probability of small $\theta$ values and because of the fast decline in the flux.

\section{Could microquasars be unidentified sources of gamma-rays detected by EGRET ?}

There is the indication that the synchrotron component of the jets in microquasars could reach the X-ray domain (Markoff, Falcke \& Fender, 2000). This would require the ejection of plasma containing electrons with Lorentz factors $\geq 10^{7}$, as in Blazars. In this context, the recent observation by Paredes et al. (2000) of persistent radio jets from LS5039, which is located in the error box of an EGRET source raises the possibility that microquasars could be persistent sources of gamma-rays.

Could microquasars be sources of $\geq 10^{20} \mathrm{eV}$ cosmic rays ?

Due to opacity by cosmic infrared photons, the sources of $\sim 10^{20}$ $\mathrm{eV}$ cosmic rays must be within $50 \mathrm{Mpc}$ from the Galaxy. AGNs have 
been proposed as possible sources of such high energy particles. As shown above, the bulk and intrinsic Lorentz factors of the electrons in microquasars are comparable to those in AGNs. Since they are much closer and numerous than the later, the very high energy cosmic rays could be produced by shocks in microquasar jets.

\section{Are Gamma-ray-bursts extreme microquasars ?}

Gamma-ray bursts are at cosmological distances and ultra-relativistic bulk motion and beaming appear as essential ingredients to solve the enormous energy requirements (Castro-Tirado et al. 1999). Beaming reduces the energy release by the beaming factor $\mathrm{f}=\Delta \Omega / 4 \pi$, where $\Delta \Omega$ is the solid angle of the beamed emission. Additionally, the photon energies can be boosted to higher values. BHs formed by core collapse producing outflows with bulk Lorentz factors $>100$ have been proposed as sources of $\gamma$-ray bursts (Mészáros \& Rees 1997). Recent studies of gamma-ray afterglows suggest that they are highly collimated jets since breaks and a steepening from a power law in time $t$ proportional to $\mathrm{t}^{-1.2}$, ultimately approaching a slope $\mathrm{t}^{-2.5}$ have been observed in light curves (Castro-Tirado et al. 1999).

It is interesting that the power laws that describe the light curves of the ejecta in microquasars show similar breaks and steepening of the radio flux density (Rodríguez \& Mirabel, 1999). In microquasars, these breaks and steepenings have been interpreted (Hjellming \& Johnston 1988) as a transition from slow intrinsic expansion to free expansion in two dimensions. Besides, linear polarizations of about $2 \%$ were recently measured in the optical afterglows, providing strong evidence that the afterglow radiation from gamma-ray bursters is, at least in part, produced by synchrotron processes. Linear polarizations in the range of $2-10 \%$ have also been measured in microquasars at radio (Rodríguez et al. 1995; Hannikainen et al. 2000), and optical (Scaltriti et al. 1997) wavelengths.

In this context, microquasars in our own Galaxy seem to be less extreme local analogs of the super-relativistic jets associated to the more distant $\gamma$-ray bursters, which are jets with Lorentz factors 2 orders of magnitude larger. Therefore, the physical mechanism that launches the jets in $\gamma$-ray bursters are likely to be different to the microquasars. Furthermore, GRBs do not repeat and seem to be related to catastrophic events, and have much larger super-Eddington luminosities. Therefore, the scaling laws in terms of the black hole mass that are valid in the analogy between microquasars and quasars do not seem to apply in the case of $\gamma$-ray bursters. 


\section{Acknowledgements}

This work was partially supported by Consejo Nacional de Investigaciones Científicas y Técnicas de Argentina.

\section{References}

Belloni, T, Méndez, M, King, AR, van der Klis, M, van Paradijs, J. 1997, Ap. J. 479: L145-48

Blandford, RD, Payne, DG. 1982, MNRAS 199: 883

Blandford, RD \& Rees, M.J. 1974, MNRAS 169, 395

Blandford, RD, Znajek, RL. 1977, MNRAS 179: 433

Bodo, G, Ghisellini, G. 1995, Ap. J. 441: L69-71

Castro-Tirado, AJ. et al. 1994, Astrophys. J. Supp. Ser. 92, 469

Castro-Tirado, AJ. et al. 1999, Science 283: 2069-73

Colbert, E.J.M. \& Mushotzky, R.F. 1999, ApJ 519, 89

Dar, A. \& De Rújula, A. 2000, astro-ph/0008474

Dhawan, V., Mirabel, I.F. \& Rodríguez, L.F. 2000, ApJ 543, 373

Eikenberry, SS, Matthews, K, Morgan, EH, Remillard, RA, Nelson, RW. 1998, Ap. J. 494: L61-64

Fender, R.P. 2000, astro-ph/0008447

Fender, RP, Pooley, GG. 1998, MNRAS 300: 573-76

Fomalont, E.B., Geldzahler, B.J. \& Bradshaw, C.F. 2000, submitted to ApJ Letters Frontera, L. et al. 2000, Proceedings of the IV Integral meeting, Alicante, Sept. 2000

Greiner, J., Morgan, E.H. \& Remillard, R.A. 1996, ApJ 473, L107

Grove, J.E. 1999, ASP Conference Series, 161, p54

Hannikainen, DC, et al. 2000, ApJ 540, 521

Hjellming, RM, Johnston, KJ. 1988, Ap. J. 328: 600-09

Junor, W., Birettta, J.A. \& Livio, M. 1999, Nature 401, 891

Kaiser, C.R., Sunyaev, R. \& Spruit, H.C. 2000, A\&A 356, 975

Livio, M. 1999, Physics Reports 311, 225

Makishima, K. et al. 2000, ApJ 535, 632

Markoff, S., Falcke, H. \& Fender, R. 2000, astro-ph/0010560

Meier, D.L, Edgington, S, Godon, P, Payne, DG, Lind, KR. 1997, Nature 388: 350-52

Meier, D.L. 2001, Review in this issue.

Mészáros, P, Rees, MJ. 1997, Ap. J. 482: L29-32

Mirabel, I.F. \& Rodríguez, L.F. 1998, Nature, 392, 673

Mirabel, I.F. \& Rodríguez, L.F. 1999, ARAA 37, 409

Mirabel, I.F. et al. 1998, A\&A 330, L9

Mirioni, L. \& Pakul, M. 2000, Private communication

Morgan, E.H., Remillard, R.A. \& Greiner, J. 1997, ApJ 482, 993

Paredes, J. M., Marti, J., Ribó, M., Massi, M. 2000, Science 288, 2340

Rodríguez, L.F. et al. 1995, Ap.J.Supp. 101, 173

Rodríguez, LF, Mirabel, IF. 1999, Astron. Astrophys. 340: L47-50

Sams, B.J., Eckart, A. \& Sunyaev, R. 1996, Nature 382, 47

Scaltriti, F. et al. 1997, A\&A 325, L29

Zhang, N.S., Cui, W. \& Chen, W. 1997, ApJ 482, L155 
mirabel_TVI-07b.tex; 29/10/2018; 22:28; p.10 